sophy from the time of Plato has had its own way of meeting it on its own ground, and disposing of its exclusive claims. I do not write here in the interests of transcendentalism, but merely to invite the attention of physicists to a point of view which students of modern psychology have borrowed from it, and are now generally seeking to apply to the problem of the relation between mental and physical energy.

Birmingham, June 9.

\section{Seismometry and Gëite.}

Had Dr. Chree (Nature, May 2I, p. 55) referred to the various papers about earthquakes in the reports of the British Association commencing in 1847 by William Hopkins, in the now somewhat antiquated Transactions of the Seismological Society of Japan, and in very many other publications relating to earthquakes, he would have seen that his instructive remarks relating to the propagation of waves in an isotropic medium were but repetitions of information with which seismologists have at least a slight acquaintance, whilst the suggestion that the velocities of such waves have been regarded as having a direct connection with Young's modulus is incorrect.

In connection with Bessemer steel, Young's modulus was mentioned, but I do not see that it was referred to repeatedly (Nature, April 9, p. 538). In 1897 Dr. Chree made an attempt to calculate Young's modulus and the bulk modulus for the earth, but the grist he used was so doubtful in character that his results are not convincing. From some source or other he discovered that wave velocities of 12.5 and $2.5 \mathrm{~km}$. per second had been determined, and these were assumed to be $V_{1}$ and $V_{2}$ for compressional and distortional waves passing through the world. One, if not both of these, are based upon arcual measurements they are incorrect at that, and the latter seens more likely to represent the velocity of a surface undulation rather than a quantity corresponding to $\mathrm{V}_{\text {. }}$.

What I pointed out was that recent determinations of a quantity probably corresponding to $V_{1}$ find a simple explanation by the assumption of a core that is fairly homogeneous and of fairly definite dimensions, which is not the solution of the seismological problems attempted by Dr. Chree. The reference to elastic moduli was incidental.

The chief objection raised to the iron core is not that iron, as we know it, will not convey vibrations at the observed speeds, but that if we take such a core, gravitational and astronomical requirements appear to be such that it must have dimensions which do not altogether accord with the interpretation given to seismometrical observations.

What Dr. Chree tells us about the possible relationship between seismic disturbances and the movements of magnetic needles is as well known to seismologists as what he has to say about wave velocities. Many of the chief magnetic observatories of the world have compared their magnetograms with long lists of world-shaking and other earthquakes, and the results are to be found in the British Association Reports, 1888 and 1889 . From Dr. Chree's own comparisons at Kew (British Association Report, 1888, pp. 229 and $23 \mathrm{r}, 8 \mathrm{cc}$ ), the movements he discovered were, with two possible exceptions, of "the ordinary magnetic small wave type," which "go on for hours if not for days." My conclusion is that at Kew, Greenwich, \&c., needles seem not to be disturbed at the time of large earthquakes in the manner in which they are disturbed at Bombay and other places. At these latter places, where the movement of needles apparently accompanying the passage of the large waves indicates a possible magnetic disturbance directly due to seismic causes, the inference I made was that at such places H.F. and $(g-\gamma)$ may be abnormal. As an illustration of the coexistence of the three phenomena we may take the following :--

$\begin{array}{lccc}\text { H.F. (c.g.s.) } & (g-y) \mathrm{cm} . & \begin{array}{c}\text { Earthquake } \\ \text { effect on } \\ \text { magnetic needles } \\ \text { Kew }\end{array} 0.1845 \mathrm{I}(\mathrm{I} 90 \mathrm{I})+40(\mathrm{IgO0}) & \begin{array}{l}\text { Undisturbed. } \\ \text { Disturbed. }\end{array}\end{array}$

Whether these coincidences are accidental or general, observations are yet required.

NO. 1754 , VOL 68$]$

\section{THE VITALITY OF THE TYPHOID BACILLUS.}

$T$ HE object of hygiene is to prevent disease. It is therefore necessary that the factors in the causation and dissemination of disease should be understood in order that adequate preventive measures may be adopted. The living agents responsible for the production of infectious diseases when they are discharged from affected individuals may find their way back to the human body by a number of indirect channels. The water, the soil, or the food may at times harbour and transmit the germs of disease. The conditions under which these morbid agents exist in the outside world constitute one of the most important subjects of hygienic inquiry. It cannot be said with regard to this phase in the life-history of pathogenic organisms that our knowledge is as accurate or extensive as it is in other directions. This is due to the difficulties that stand in the way of such investigations. The germs of disease undergo an enormous dilution in the air, water and soil, whilst they tend to become lost in the crowd of similar forms already existing in nature. The facts so far support the view that the parasitic microorganisms possess a considerable amount of resistance to external influences, and that the links which ensure their conservation and retransference to man are numerous and varied. A typical example is the bacillus of typhoid fever. This organism may become widely distributed through the dejecta. It may contaminate a water supply and directly, or by the agency of milk, produce a fresh outbreak of typhoid fever. It may infect the soil, and through it a number of raw vegetable foods. Its presence has been detected in the sewage-fed oyster, whilst tainted dust and flies aid in the distribution of the organism.

In studying the distribution of enteric fever, a physical factor which has to be considered is the influence of cold on the vitality of the specific organism. The effect of low temperatures upon microorganisms generally has formed a subject of inquiry from time to time. The latest experimental work has conclusively shown that bacteria retain their vitality under the most adverse conditions of cold that it is possible to devise. Prof. Sedgwick and Mr. Winslow, approaching the subject from the hygienic point of view, have carefully studied the influence of natural and normal conditions of cold upon the typhoid bacillus in particular. Their experiments were carried out with special reference to the danger of conveyance of the disease in question by polluted ice, and with reference to the seasonal distribution of the disease. The matter was undoubtedly one that called for investigation, and notably in a country where ice and iced drinks are in such universal demand. The authors were unable to find any recorded evidence of a conclusive character as to the spread of typhoid fever by a polluted ice supply, although it has been a common opinion that ice might be an important source of infection for typhoid fever and other intestinal diseases.

The apparent purity of ice is deceptive. It is true that water in freezing undergoes a certain amount of purification. It loses, on conversion into ice, saline constituents, contained air, and a certain proportion of organic suspended matter. At the same time, it is not entire!y freed from microbes. The figures quoted by Prof. Sedgwick and Mr. Winslow show that snow-ice may contain an average of more than 600 bacteria per cub. cm. Figures are also given to indicate the enor-

1 Experiments on the Effect of Freezing and other Low Temperatures upon the Viability of the Bacillus of Typhoid Fever, with Considerations regarding Ice as at Vehicle of Infectious Disease "By William $T$. Sedgwick, Ph.D., Professor of Biology, and Charles-Edward A. Winslow, S. M. Instructor in Biology in the Massachusetts Institute of Technology (Memoirs of the American Academy of Arts and Sciences, vol. xii. No. 5 1902.) 
mous number of bacteria present at times in ice-creams - one of the highest records being seven millions in one cub. cm. The sources of danger in ice-creams are obvious, as they come from the spoons and vessels, and the persons and dwellings of the street vendors.

Laboratory experiments have confirmed the conclusion that a freezing process is not necessarily fatal to bacterial life. We have instances of bacteria multiplying at zero, and of their survival after a six months' exposure to the temperature of liquid air. It is not therefore surprising that the American observers were unable to secure a complete sterilisation of bacterial cultures by the freezing methods they employed. The question became therefore a relative one. What was the probability or likelihood of infection through ice in the case of typhoid fever? It would appear that about 90 per cent. of the ordinary water bacteria are eliminated by the process of freezing. The authors find that, in the case of a specific pathogenic organism such as the Bacillus typhosus, less than one per cent. survive simple freezing for a period of fourteen days. Complete sterility did not occur even at the end of three months, whilst a process of alternate thawing and freezing, if on the whole more fatal to the typhoid germs than a simple freezing, was equally unsuccessful in effecting an absolute sterilisation of the infected water. The reduction in the number of typhoid bacilli in chilled water was approximately as great as occurred in ice. The process of destruction proved to be a continuous one, whether it occurred above or below the freezing-point, and whether the experiments were made in water or in soil. A progressive reduction in the number of organisms occurred to the extent of about 99 per cent., and proceeded pari passu with the duration of the experiment. Cold exercises a disinfecting action as regards the typhoid bacillus, and in natura? ice there is a supplementary purifying influence to be taken into account, as, at the time of freezing, 90 per cent. of the germs are thrown out by a process of physical exclusion. These are the main conclusions arrived at, and the authors find that they are in accord with the general result of experience, namely, that natural ice can very rarely be a vehicle of typhoid fever.

The research may perhaps fairly be described as a study of the death-rate of typhoid bacilli under adverse conditions, as furnished by cold. The percentage mortality, as a matter of fact, is such as might occur under the influence of light, a poor food supply, and disinfectant agents generally. It is therefore permissible to think that the danger of infection in the case of ice, if it is minimised, is not by any means abolished. A certain number of typhoid bacilli, as the experiments show, do remain alive, and these may, on rethawing, undergo a rapid multiplication outside as well as inside the human body. And it has likewise to be remembered that it is notoriously difficult to trace the exact channels of infection in sporadic cases of typhoid fever. The infection has at times occurred from the most unexpected quarters.

Prof. Sedgwick and Mr. Winslow have rightly drawn attention to the unfavourable conditions furnished by natural ice for the propagation of the typhoid organism. It is at the same time feasible to assume that ice may likewise act as a conserving agent, inasmuch as the cold, whilst inhibiting the growth of the typhoid bacillus, will equally prevent the multiplication of other competitive forms of life.

The experiments do not affect the general question of the persistence of life at low temperatures. If the temperature be sufficiently low to produce a complete anæsthesia of the cells, cold tends to act as a conserving agent on the typhoid bacillus and allied forms.

It only remains to commend the memoir of Prof.
Sedgwick and Mr. Winslow to the attention of all who are interested in the epidemiological questions involved.

Allan Macfadyen.

\section{NOTE ON THE PROBABLE OCCASIONAL INSTABILITY OF ALL MATTER.}

$A \mathrm{~S}$ a summary of my remarks at the discussion on A Prof. Rutherford's most interesting communication on the subject of radio-activity to the Physical Society of London on Friday last, June 5, I beg to communicate the following :-

Consider an electron or other particle, of mass $m$ and of negative charge $e$, revolving at speed $u$ round the much more massive rest of an atom possessing an equal positive charge. The centripetal force between them is

$$
\stackrel{m u^{2}}{r}=\frac{e^{2}}{\mathrm{~K} r^{2}}
$$

where the first $r$ strictly is measured to the centre of gravity of the two bodies, and the second $r$ is the distance between their centres; but taking these as usual practically equal for the lighter body, we get Kepler's law for the case

$$
r u^{2}=\text { const. }=\frac{e^{2}}{\mathrm{~K} m}
$$

Larmor has shown (" Fther and Matter," p. 227) that an electric charge subject to acceleration radiates some of its kinetic energy, though the radiation becomes of prominent amount only when the acceleration is great; as, for instance, when kathode rays are suddenly stopped by a target. The "power" of the radiation, or the energy lost per unit time, is

$$
\mathrm{R}=\frac{2 \mu e^{2} \dot{i}^{2}}{3 v}
$$

where $u$ is the acceleration of the electric charge $e$, and $v$ is the velocity of light.

In the case of steady circular motion, the only acceleration is normal or centripetal, viz.

$$
\dot{u}=u \iota^{2} / r
$$

but that is just as effective for radiation purposes as the tangential variety.

Hence, combining the three equations, we get, for the radiating power,

$$
\mathrm{R}=\frac{2}{3 \mu} \cdot\left(\frac{m}{e}\right)^{2} \cdot \frac{u^{8}}{v^{5}}
$$

that is, a constant multiplied by the eighth power of the velocity of the rapidly moving particle: an expression which corresponds with what for ordinary molecular motions is known as Stefan's law, connecting radiation with temperature, i.e. with square of molecular velocity.

Now the radiation loss is equivalent to a resisting medium, and accordingly the revolving particle tends to move inwards towards its centre, and its speed to increase in accordance with equation (r). A slight increase in speed brings about a great increase in radiating power, as is shown by equation (4); wherefore the change, once appreciably begun, may be expected to go on rapidly, until presently the speed approaches the velocity of light. On the electric theory of matter, radiation or loss of energy must occur from every atom, and therefore it is only a question of time how long an atom shall last before it reaches this stage.

Directfy this stage is reached another effect supervenes; the rapidly moving portion of the mass begins rapidly to rise in value, according to a complicated expression not yet quite fully worked out. This effect

NO. 7754 , VOL. 69$]$ 